\title{
Key performance indicators score (KPIs-score) based on clinical and laboratorial parameters can establish benchmarks for internal quality control in an ART program
} \author{
Baruffi, ${ }^{1,2}$ \\ ${ }^{1}$ Center for Human Reproduction Prof. Franco Jr., Ribeirao Preto, Brazil \\ ${ }^{2}$ Paulista Center for Diagnosis, Research and Training, Ribeirao Preto, Brazil
}

José G. Franco Jr ${ }^{1,2}$, Claudia G. Petersen ${ }^{1,2}$, Ana L. Mauri ${ }^{1,2}$, Laura D. Vagnini ${ }^{2}$, Adriana Renzi ${ }^{2}$, Bruna Petersen ${ }^{2}$, M.C. Mattila ${ }^{1}$, Vanessa A. Comar ${ }^{1}$, Juliana Ricci ${ }^{1}$, Felipe Dieamant ${ }^{1,2}$, João Batista A. Oliveira ${ }^{1,2}$, Ricardo L.R.

\begin{abstract}
Objective: KPIs have been employed for internal quality control (IQC) in ART. However, clinical KPIs (C-KPIs) such as age, AMH and number of oocytes collected are never added to laboratory KPIs (L-KPIs), such as fertilization rate and morphological quality of the embryos for analysis, even though the final endpoint is the evaluation of clinical pregnancy rates. This paper analyzed if a KPIs-score strategy with clinical and laboratorial parameters could be used to establish benchmarks for IQC in ART cycles.
\end{abstract}

Methods: In this prospective cohort study, 280 patients (36.4 4 .3years) underwent ART. The total KPIsscore was obtained by the analysis of age, AMH (AMH Gen II ELISA/pre-mixing modified, Beckman Coulter Inc.), number of metaphase-II oocytes, fertilization rates and morphological quality of the embryonic lot.

Results: The total KPIs-score (C-KPIs+L-KPIs) was correlated with the presence or absence of clinical pregnancy. The relationship between the C-KPIs and L-KPIs scores was analyzed to establish quality standards, to increase the performance of clinical and laboratorial processes in ART. The logistic regression model (LRM), with respect to pregnancy and total KPIs-score (280 patients $/ 102$ clinical pregnancies), yielded an odds ratio of $1.24(95 \% C I=1.16-1.32)$. There was also a significant difference $(p<0.0001)$ with respect to the total KPIs-score mean value between the group of patients with clinical pregnancies (total KPIs-score $=20.4 \pm 3.7$ ) and the group without clinical pregnancies (total KPIs-score $=15.9 \pm 5$ ). Clinical pregnancy probabilities (CPP) can be obtained using the LRM (prediction key) with the total KPIs-score as a predictor variable. The mean C-KPIs and L-KPIs scores obtained in the pregnancy group were $11.9 \pm 2.9$ and $8.5 \pm 1.7$, respectively. Routinely, in all cases where the C-KPIs score was $\geq 9$, after the procedure, the L-KPIs score obtained was $\leq 6$, a revision of the laboratory procedure was performed to assess quality standards.

Conclusion: This total KPIs-score could set up benchmarks for clinical pregnancy. Moreover, IQC can use C-KPIs and L-KPIs scores to detect problems in the clinicallaboratorial interface.

Keywords: KPI, internal quality control, clinical pregnancy, IVF, ICSI, AMH

\section{INTRODUCTION}

Systems to monitor clinical and laboratorial performance have gained much importance in medical practice (Leandro et al., 2005; Vermeulen et al., 2008; Salinas et al., 2010). The assessment of clinical or surgical practices should take advantage of techniques developed for controlling processes in the manufacturing industry.

A performance indicator or key performance indicator (KPI) is a type of performance measurement. Any process, whether in a biomedical or non-biomedical field, can be subject to inherent deviations from the optimum or from established limits. These deviations may lead to defective end-products or, in the medical field, defective patient care. Monitoring, which is a process able to identify deviations, and then being able to act should such deviation exceed certain limits, plays an important role in avoiding adverse consequences and maintaining optimal performance.

It should be noted that published studies (PubMed research) about KPIs and ART are practically nonexistent. Information is usually obtained through classes on laboratorial quality control, or opinions of authors expressed in chapters of books. In this way, KPIs have been employed for internal quality control (IQC) in IVF/ICSI programs, using indicators such as oocyte fertilization, cleavage embryo rates, percentage of topquality embryos, etc. KPIs analyses can be plotted and compared with established limits for the mean and standard deviation values, so that deviations can be easily recognized as warnings or action points, but possibility a false interpretation of a KPI drop could not be excluded. Laboratory conditions (temperature, $\mathrm{pH}$, humidity, air quality, culture media, equipment, etc.) could modify these variables and negatively impact the outcomes. Since the KPIs data is available after some weeks, the impact of a problem not detected in the laboratory may potentially affect outcome for a significant period.

However, in no time, clinical KPIs (C-KPIs) such as age, AMH and number of oocytes collected are added to laboratory KPIs (L-KPIs) for analysis, even though the final endpoint is the evaluation of clinical pregnancy rates.

The purpose of this study was to develop a total KPIs-score (C-KPIs+L-KPIs) with the power to identify individual benchmarks, as well as to analyze the laboratory performance during different situations. The KPIs scores strategy application could result in an immediate evaluation of the patient's clinical and laboratory performance in the ART cycle. In addition, internal quality control benchmarks could be evaluated.

\section{MATERIALS AND METHODS}

Patients' characteristics and inclusion criteria

In this prospective cohort study, 280 patients (36.4 4.3years) were submitted to ICSI cycles during 2015-2016. All patients met the following criteria: body mass index (BMI) between $20-30 \mathrm{~kg} / \mathrm{m}^{2}$, regular menstrual cycles, both ovaries present, no history of ovarian surgery, no severe endometriosis and no evidence of endocrine disorders. The study was authorized by the local ethical committee, and a written informed consent was obtained from all recruited subjects.

The KPIs score was obtained by analysis of age, $\mathrm{AMH}$ $(\mathrm{ng} / \mathrm{ml})$, number of metaphase-II oocytes, fertilization rates and morphological quality of embryonic lot (MQEL). 
The maximum total KPIs score was 25 points (age $\leq 36$, AMH $\geq 2$, number of oocytes on metaphase II $\geq 7$, fertilization rate $\geq 65 \%$, number of top quality embryos $\geq 2$ ), the minimum total KPIs score was 5 points (age $\geq$ 40 , $\mathrm{AMH}<1$, number of oocytes on metaphase II $\leq 3$, fertilization rate $<50 \%$, only low quality embryos (see for more details: Figure 1).

\section{AMH measurement}

A venous blood sample for an AMH measurement was taken before the scheduled treatment (minimum of 30 days), during the early follicular menstrual cycle phase in all the women. AMH was measured using an enzymatically amplified 2-site immunoassay kit (AMH Gen II ELISA/ modified, Beckman Coulter Inc.). These AMH levels were obtained after modifying the methodology suggested by Han et al. (2014). The lowest detection limit of this assay is $0.01 \mathrm{ng} / \mathrm{ml}$, whereas the maximum intra- and inter-assay variation coefficients were $3.3 \%$ and $6.5 \%$, respectively. To minimise the likelihood of bias in the assay, all sera were processed in duplicate during the same day, using the same measurement kits, and it was done by the same operator. Low- and high-level controls were included in each assay.

\section{Ovarian stimulation protocol}

The patients were subjected to 2 schemes of controlled ovarian stimulation:

I - GnRH-agonist protocol: the pituitary downregulation began during the luteal phase of the previous menstrual cycle with $\mathrm{GnRH}-\mathrm{a}$ leuprolide acetate (leuprolide acetate; Lupron ${ }^{\circledR}$; Abbott, Brazil) at a dose of $1 \mathrm{mg} /$ day for 14 days. The ovaries were then stimulated with a fixed dose of 75-375IU of recombinant FSH ( $r-F S H$; Gonal F ${ }^{\circledR}$; Serono, Brazil) and 75IU/day of recombinant luteinising hormone ( $\mathrm{r}$-LH; Luveris $^{\circledR}$; Serono, Brazil) for a period of 7 days. On day 8 of the ovarian stimulation, follicular development was monitored by a transvaginal ultrasound at $7 \mathrm{MHz}$. The r-FSH dose was modified according to the ovarian response, and $r$-LH supplementation was increased to 150 IU/day when one or more follicles measuring $\geq 10 \mathrm{~mm}$ in diameter were found.

II - GnRH-antagonist protocol: On day 3 of the cycle, ovarian stimulation was induced with a fixed dose of 75$375 \mathrm{IU}$ of $\mathrm{r}-\mathrm{FSH}$ and 75IU/day of r-LH for a period of 5 days. On day 8 of the menstrual cycle (day 6 of ovarian stimulation), the follicular development was monitored by transvaginal ultrasound at $7 \mathrm{MHz}$. The r-FSH dose was modified according to the ovarian response, and the $\mathrm{r}$ - $\mathrm{LH}$ supplementation was increased to $150 I U /$ day when 1 or more follicles measuring $\geq 10 \mathrm{~mm}$ in diameter were found. The GnRH-ant (cetrorelix; Cetrotide ${ }^{\circledR}$; Serono, Brazil) was started at a dose of $0.25 \mathrm{mg} /$ day s.c., when at least 1 follicle of $\geq 14 \mathrm{~mm}$ was seen in the ultrasound scan.

To induce final oocyte maturation in both protocols

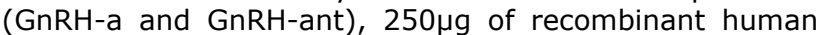
chorionic gonadotropin ( $\mathrm{r}$-hCG; Ovidrel; Serono, Brazil) was administered S.C., when at least 2 follicles reached a mean diameter of $\geq 17 \mathrm{~mm}$. Except at the risk of developing SHO when GnRH-a was added at 1500IU doses of hCG for final oocyte maturation. GnRH-a and GnRH-ant were administered until the day of the r-hCG injection. Oocyte retrieval was performed by transvaginal aspiration under ultrasound guidance 34-36 hours following the r-hCG injection.

\section{Metaphase II oocytes}

The retrieved oocytes were incubated in culture medium for $1-2$ hour(s). Cumulus cells were removed by exposing the oocytes to hyaluronidase, after which coronal cells were removed and the denuded oocytes were classified according to their level of maturation. Oocytes with the first polar body, i.e., in metaphase II (MII) were considered to be mature and the total number used in ICSI was used as a benchmark for the KPIs-score.

\section{Fertilization rates}

Fertilization was seen $16-19 \mathrm{~h}$ after the procedure, to determine the presence or absence of pronuclei. A normal fertilization process was defined on the basis of the formation of two distinct pronuclei. The percentage of fertilized oocytes was used as benchmark for the KPIsscore.

\section{Morphological quality of the embryonic lot}

The morphological quality of the embryonic lot was evaluated routinely from 48 to 72 after injection at the cleavage stage, depending on the day of transfer, day 2 or day 3 , respectively.

Embryos transferred at day 2: They were scored according to the following criteria:

-Grade 6 (top quality): embryos presenting 4 cells (blastomeres), with symmetrical blastomeres, with no blastomere fragmentation and with one nucleus in each blastomere;

-Grade 5 (top quality): embryos presenting 4 cells (blastomeres), with symmetrical blastomeres, with no blastomere fragmentation and absence of one nucleus in each blastomere;

-Grade 4 (intermediary quality): embryos without 4 cells (blastomeres), with symmetrical blastomeres and with no blastomere fragmentation;

-Grade 3: embryos presenting 4 cells (blastomeres), with irregular blastomeres and with $\geq 10<25 \%$ of blastomere fragmentations;

-Grade 2: embryos presenting 4 cells (blastomeres), with irregular blastomeres, with $\geq 25 \%$ of blastomere fragmentations;

-Grade 1: embryos without 4 cells (blastomeres), with irregular blastomeres, with $\geq 10<25 \%$ of blastomere fragmentations;

-Grade 0: embryos without 4 cells (blastomeres), with irregular blastomeres, with $\geq 25 \%$ of blastomere fragmentations.

Embryo transferred on day 3: They were scored per the following criteria:

-Grade 5 (top quality): embryos presenting 8 cells (blastomeres), with symmetrical blastomeres and with no blastomere fragmentation.

-Grade 4 (intermediary quality): embryos without 8 cells (blastomeres), with symmetrical blastomeres and with no blastomere fragmentation.

-Grade 3: embryos presenting 8 cells (blastomeres), with irregular symmetrical blastomeres and with $\geq 10<$ $25 \%$ blastomere fragmentation.

-Grade 2: embryos presenting 8 cells (blastomeres), with irregular symmetrical blastomeres and with $\geq 25 \%$ blastomere fragmentation.

-Grade 1: embryos without 8 cells (blastomeres), with irregular symmetrical blastomeres and with $\geq 10<25 \%$ blastomere fragmentation.

-Grade 0: embryos without 8 cells (blastomeres), with irregular symmetrical blastomeres and $\geq 25 \%$ blastomere fragmentation.

\section{KPI points evaluation:}

-5 points $=$ the embryonic lot presented $\geq 2$ embryos scored Grade 6 or Grade 5;

-3 points $=$ the embryonic lot presented 1 embryo scored Grade 6 or Grade 5, or 2 embryos scores Grade 4; 


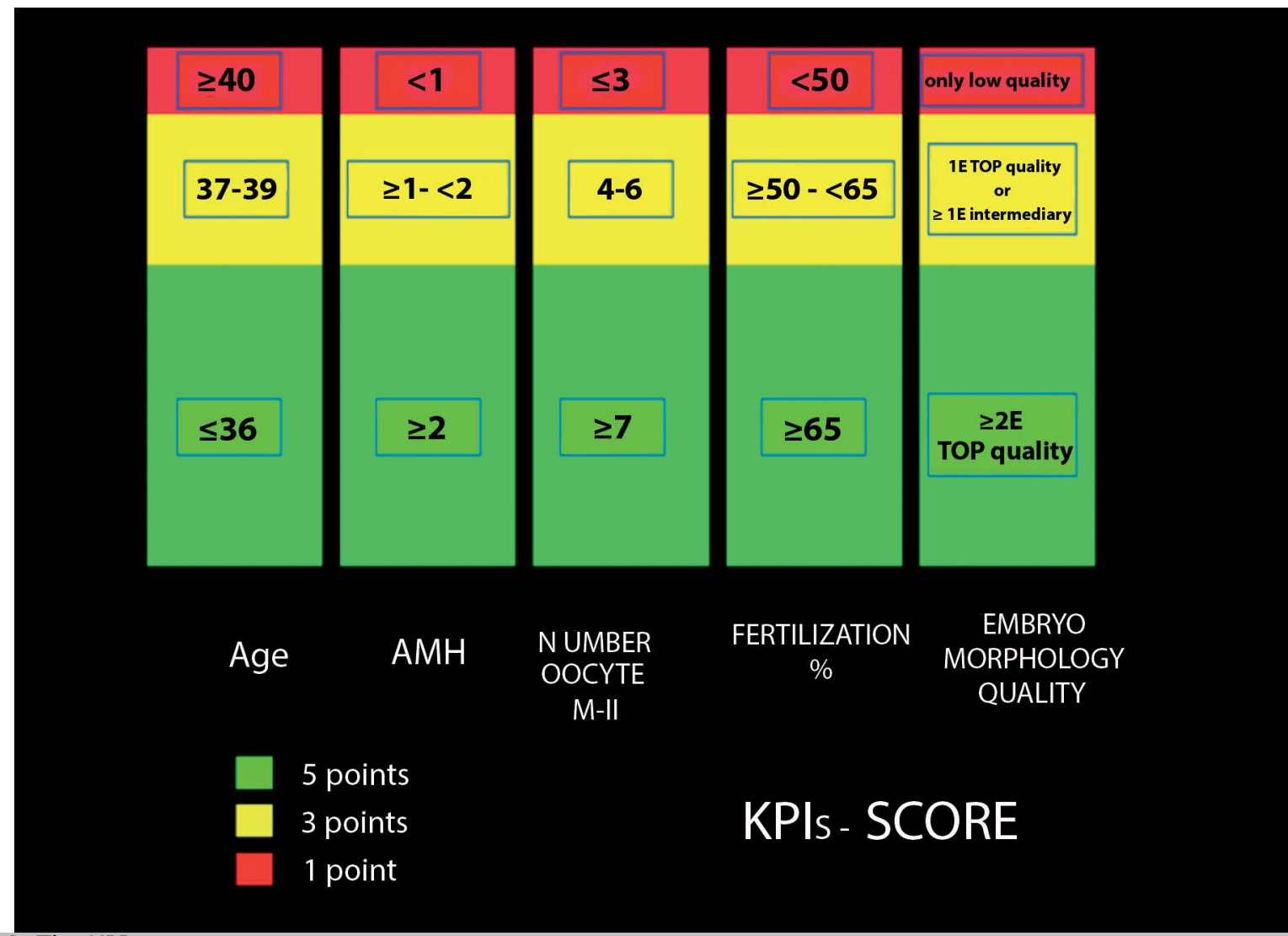

Figure 1. The KPIs- score system.

-1 point $=$ the embryonic lot presented only embryos Grade $3,2,1,0$ or 1 embryo Grade 4 .

Clinical pregnancy: Clinical pregnancy was defined as the presence of a gestational sac in the uterine cavity with a heartbeat at 6 gestation's week, detected by ultrasonography.

Statistical analysis: The data was analyzed using the Stats Direct statistical software (Cheshire, UK). For dichotomous variables, correlations were performed using the logistic regression model (LRM).

\section{RESULTS}

\section{Clinical pregnancy benchmark}

The total KPIs-score (C-KPIs+L-KPIs) was correlated with the presence or absence of clinical pregnancy. The relationship between the C-KPIs score and L-KPIs score was analyzed to establish quality standards to increase the performance of the clinical and laboratory work in ART.

The logistic regression model with respect to clinical pregnancy and total KPIs-score (280 patients/102 clinical pregnancy) yielded an odds ratio of 1.24 (95\%CI $=1.16$ $1.32)$. Also, there was a significant difference $(p<0.0001)$ with respect to the total KPIs mean score among the group of patients with clinical pregnancy (total KPIsscore $=20.4 \pm 3.7$ ) and the group without clinical pregnancy (total KPIs-score $=15.9 \pm 5$ ). Clinical pregnancy probabilities (CPP) can be obtained using the LRM (prediction key) with the total KPIs-score as a predictor variable; therefore, total KPIs-score $25 / \mathrm{CPP}=70 \%(95 \% \mathrm{CI}=59 \%-79 \%)$; total KPIsscore $=20 / \mathrm{CPP}=45 \% \quad(95 \% \mathrm{CI}=38 \%-51 \%)$, total KPIsscore $=15 / \mathrm{CPP}=22 \% \quad(95 \% \mathrm{CI}=16 \%-30 \%)$, total KPIsscore $=10 / C P P=9 \%(95 \% C I=5 \%-15 \%)$, etc. (Table 1$)$.

\section{C-KPIs and L-KPIs benchmarks}

On the other hand, the mean C-KPIs score and L-KPIs scores obtained in the pregnancy group were $11.9 \pm 2.9$ and $8.5 \pm 1.7$, respectively. Routinely, in all cases in which the C-KPIs score was $\geq 9$ after the procedure but the L-KPIs score obtained was $\leq 6$ (approximately one standard deviation and half less than the mean value), a revision of the laboratory procedure was performed to check the quality standards.

\section{DISCUSSION}

The KPIs system was used to detect early warning signals in gamete/embryo cultures. However, the final endpoint is the evaluation of clinical pregnancy rates, although the C-KPIs are not usually included in the final evaluation.

In this study, the C-KPIs was a product of the analysis of three variables that have a significant correlation with pregnancy rates (age, AMH and number of oocytes collected).

C-KPIs Predictor variable/Age: age is the most traditional factor negatively correlated with pregnancy and live birth rates (LBR) after IVF/ICSI. This association between female age and LBR was nonlinear, with marked decreases in LBR after 28, 35 and 38 years of age (Vaegter et al., 2017). Some studies dichotomized age into two categories, $<35$ or $\geq 35$ years (Sharma et al., 2002). Women aged 35 years or older had significantly lower pregnancy rates when compared with women who were younger than 35 years. Others studies categorized the patients into four groups, i.e., 30, 30-34, 35-38 and 39- 
Table 1. Total KPIs-score and clinical probabilities

\begin{tabular}{|c|c|c|}
\hline $\begin{array}{l}\text { TOTAL } \\
\text { KPIs-SCORE }\end{array}$ & $\begin{array}{c}\text { CLINICAL } \\
\text { PREGNANCY } \\
\text { PROBABILITIES }\end{array}$ & $\begin{array}{l}\text { CONFIDENCE } \\
\text { INTERVAL }\end{array}$ \\
\hline 25 & $70 \%$ & $59 \%-79 \%$ \\
\hline 24 & $65 \%$ & $56 \%-74 \%$ \\
\hline 23 & $60 \%$ & $51 \%-69 \%$ \\
\hline 22 & $55 \%$ & $47 \%-63 \%$ \\
\hline 21 & $50 \%$ & $42 \%-57 \%$ \\
\hline 20 & $45 \%$ & $38 \%-51 \%$ \\
\hline 19 & $40 \%$ & $33 \%-46 \%$ \\
\hline 18 & $35 \%$ & $28 \%-41 \%$ \\
\hline 17 & $30 \%$ & $24 \%-36 \%$ \\
\hline 16 & $26 \%$ & $20 \%-32 \%$ \\
\hline 15 & $22 \%$ & $16 \%-30 \%$ \\
\hline 14 & $18 \%$ & $13 \%-26 \%$ \\
\hline 13 & $15 \%$ & $10 \%-22 \%$ \\
\hline 12 & $13 \%$ & $8 \%-20 \%$ \\
\hline 11 & $10 \%$ & $6 \%-18 \%$ \\
\hline 10 & $9 \%$ & $5 \%-15 \%$ \\
\hline 9 & $7 \%$ & $4 \%-14 \%$ \\
\hline 8 & $6 \%$ & $3 \%-12 \%$ \\
\hline 7 & $5 \%$ & $2 \%-10 \%$ \\
\hline 6 & $4 \%$ & $1.6 \%-9 \%$ \\
\hline 5 & $3 \%$ & $1.2 \%-8 \%$ \\
\hline
\end{tabular}

45 years (Sabatini et al., 2008). Women among the 30 and 30-34 years age-categories had 3.2 and 2.8 higher pregnancy likelihood when compared with women in the age category of 39-45 years. The third study showed that women aged 30 years or older had lower pregnancy rates when compared with women in the 25-29 group (Wang et al., 2008). The biological explanation for this decline in conceiving likelihood with increasing female age most likely lies in the diminished ovarian reserve, a decrease in both quantity and quality of oocytes, which is clinically relevant in women from their mid-30s (Broekmans et al., 2007). Despite difficulties in establishing the ideal and precise intervals of age as a benchmark in the prediction of clinical pregnancy, this study used three categories $(\leq 36$, $37-39, \geq 40$ years), almost like those described by Sabatini et al. (2008).

C-KPIs Predictor variable/AMH: Attempts have also been made to correlate serum AMH levels with the occurrence of pregnancy in ART cycles. Values higher than $2.7 \mathrm{ng} / \mathrm{mL}$ were associated with higher rates of implantation and pregnancy (Silberstein et al., 2006). Also, the level of AMH is associated with LBR after IVF/ICSI in women with (extremely) low ovarian reserve. The LBR in women with $\mathrm{AMH}>0.4 \mathrm{ng} / \mathrm{ml}$ was significantly higher than in women with AMH $\leq 0.4 \mathrm{ng} / \mathrm{ml}$ (Reijnders et al., 2016). AMH could serve as a tool in the pre-treatment counseling for pregnancy likelihood in women with (extremely) low ovarian reserve. Gleicher et al. (2016) described some relationship between levels of AMH and LBR: best LBR values (43-47\%) were obtained at AMH 3.5-7.0ng/ml; intermediate rates (32-41 $\%)$ at $\mathrm{AMH}$ of $1.5-3.0$ and $7.5-9.0 \mathrm{ng} / \mathrm{ml}$, but even poor prognosis with $\mathrm{AMH}$ of $\leq 1.0 \mathrm{ng} / \mathrm{ml}$ was still associated with
$25-29 \%$ live births. The low $(<1)$, intermediate $(\geq 1-<2)$ and high $(\geq 2) \mathrm{AMH}$ levels were defined as ranges for this C-KPIs score benchmark.

C-KPIs Predictor variable/Number of oocytes retrieved: Six studies reported on the association between the number of oocytes retrieved and pregnancy rates (Strandell et al., 2000; Hart et al., 2001; Hunault et al., 2002; Sharma etal., 2002; Ottosen etal., 2007). Two studies categorized the data. One study dichotomized the number of oocytes into $\leq 5$ and $>5$ oocytes retrieved (Sharma et al., 2002). The other study used three categories: 1-5 oocytes, 6-10 and 11 or more oocytes (Ottosen et al., 2007). Both studies found that women with more oocytes had higher likelihoods of pregnancy. Van Loendersloot et al. (2010) found a positive association between increasing number of oocytes retrieved and pregnancy likelihood after IVF, with an OR of 1.04 (95\%CI=1.02-1.07). The number of oocytes in metaphase II $(\geq 7)$ was used as the best benchmark value, corresponding to values equal to or above the mean of those previously found in the patients who achieved clinical pregnancies in our clinic.

On the other hand, L-KPIs scores were associated with two variables (fertilization rate and the morphological quality of embryos):

L-KPIs Predictor variable/Fertilization rate: The fertilization rate of the oocyte is a KPI traditionally used in monthly, semi-annual and even annual IQC evaluations in ART. However, there are no studies defining abnormal situations and what measures were adopted to solve the problem. On the other hand, one, two, or three standard deviations from an average of ideal values are used to define this benchmark. However, this KPI is not directly linked to the prediction of clinical pregnancy and, consequently, of live births in ART; but it provides basic information on laboratory quality control. The percentage of fertilization $(\geq 65 \%)$ used as best benchmark, corresponded to values equal to or above the mean of those previously observed in the patients who achieved clinical pregnancies in our clinic.

L-KPIs Predictor variable/Morphological quality of embryonic lot: Three studies evaluated the association between embryo quality and pregnancy rates after IVF (Strandell et al., 2000; Hunault et al., 2002; Ottosen et al., 2007). One study classified embryo quality using two separate factors, evaluating the best and the second-best embryos in terms of stage of development and morphology score (Hunault et al., 2002). The stage of development was described using three categories: Delayed, Appropriate and Advanced. The Advanced Stage was used as the reference category. Women in whom either the best or secondbest embryo had a delayed or appropriate development had lower pregnancy likelihood when compared with women in whom either the best or second-best embryo was at an Advanced Stage of development. Lower morphology scores were also associated with lower pregnancy likelihoods. The second study reported that women with embryos at a higher developmental stage and morphology scores, combined into one predictor, had higher pregnancy likelihoods when compared with women at lower developmental stage and morphology score (Ottosen et al., 2007). The third study used three other predictors for embryo quality: number of good quality embryos available, number of good quality embryos transferred and number of embryos suited for freezing (Strandell et al., 2000). All three predictors were associated with higher pregnancy likelihoods after IVF. In all the studies, better embryo quality was associated 
with higher likelihoods of pregnancy, but since these studies used different factors or combinations of embryo factors to report embryo quality, it was not possible to pool the data and calculate a summary OR (Vaegter et al., 2017). The number of embryos top $\geq 2$ used as best benchmark corresponded to values equal to or higher than the mean of those found previously in the patients who achieved clinical pregnancies in our clinic.

Application of the KPIs-score strategy: The data from this study showed that the values of the total KPIsscore have an excellent correlation with the rates of clinical gestation, thus becoming an excellent predictor of the cycle analyzed. In this way, it is possible to establish an individualized prognosis for each patient, avoiding a prediction based on global gestational data obtained in the clinic. On the other hand, if there is no gestation, all efforts should be directed to an increase of the total KPIsscore in a new attempt. From the clinical point of view, the main suggestions should be directed to the models of ovarian stimulation, since the age and the $\mathrm{AMH}$ values are not susceptible to modifications. However, the isolated or comparative observation of C-KPIs and L-KPIs scores also provides important information for planning an upcoming ART cycle. When C-KPIs and L-KPIs scores are maximum but gestations do not occur, other causal factors could be investigated, such as difficulties in embryo transfer processes, presence of factors that would compromise endometrial receptivity or suspected problems in luteal phase supplementation. In the situation where the C-KPIs score is $\geq 9$ and the L-KPIs score is $\leq 6$ points, an immediate laboratory evaluation should be performed for the identification or not, of future laboratory problems. At that time, an analysis of the quality of gametes is advised, as well as a detailed discussion about possible changes introduced in the laboratory (new culture media, changes in the incubator system, culture plates, embryo transfer catheters, etc.). On the other hand, there are cases where the C-KPIs score was low and the L-KPIs score reached 10 points, the term employed is positive inversion, in which case the embryologist's job should be highlighted. However, concerns about embryologists' performance can be raised when an embryologist does not exceed the L-KPIs benchmark score 6 (when the C-KPIs score benchmark is always $\geq 9$ ). This alarm signal can be used as an important instrument in the laboratory IQC. On the other hand, one of the limitations of the application of the KPIs-score strategy is that each clinic should establish its own key performance indicators and benchmarks.

\section{CONCLUSION}

This total KPIs-score could set up benchmarks for clinical pregnancy. Moreover, IQC can use C-KPIs and L-KPIs scores to detect problems in the clinical-laboratorial interface.

\section{REFERENCES}

Broekmans FJ, Knauff EA, te Velde ER, Macklon NS, Fauser BC. Female reproductive ageing: current knowledge and future trends. Trends Endocrinol Metab. 2007;18:58-65. PMID: 17275321 DOI: $10.1016 / j . t e m .2007 .01 .004$

Gleicher N, Kushnir VA, Sen A, Darmon SK, Weghofer A, Wu YG, Wang Q, Zhang L, Albertini DF, Barad DH. Definition by $\mathrm{FSH}, \mathrm{AMH}$ and embryo numbers of good, intermediate- and poor-prognosis patients suggests previously unknown IVF outcome-determining factor associated with AMH. J Transl Med. 2016;14:172. PMID: 27286817 DOI: 10.1186/s12967-016-0924-7
Han X, McShane M, Sahertian R, White C, Ledger W. Premixing serum samples with assay buffer is a prerequisite for reproducible anti-Mullerian hormone measurement using the Beckman Coulter Gen II assay. Hum Reprod. 2014;10428. PMID: 24626805 DOI: 10.1093/humrep/deu050

Hart R, Khalaf $Y$, Yeong CT, Seed P, Taylor A, Braude P. A prospective controlled study of the effect of intramural uterine fibroids on the outcome of assisted conception. Hum Reprod. 2001;16:2411-7. PMID: 11679530 DOI: http://dx.doi.org/10.1093/humrep/16.11.2411

Hunault CC, Eijkemans $M J$, Pieters $M H$, te Velde ER, Habbema JD, Fauser BC, Macklon NS. A prediction model for selecting patients undergoing in vitro fertilization for elective single embryo transfer. Fertil Steril. 2002;77:725-32. PMID: 11937124 DOI: http://dx.doi.org/10.1016/S0015-0282(01)03243-5

Leandro G, Rolando N, Gallus G, Rolles K, Burroughs AK. Monitoring surgical and medical outcomes: the Bernoulli cumulative SUM chart. A novel application to assess clinical interventions. Postgrad Med J. 2005;81:647-52. PMID: 16210461 DOI: $10.1136 / p g m j .2004 .030619$

Ottosen LD, Kesmodel U, Hindkjaer J, Ingerslev $\mathrm{HJ}$. Pregnancy prediction models and ESET criteria for IVF patients-do we need more information? J Assist Reprod Genet. 2007;24:29-36. PMID: 17165151 DOI: $10.1007 / \mathrm{s} 10815-006-9082-9$

Reijnders IF, Nelen WL, IntHout J, van Herwaarden $A E$, Braat DD, Fleischer $\mathrm{K}$. The value of Anti-Müllerian hormone in low and extremely low ovarian reserve in relation to live birth after in vitro fertilization. Eur J Obstet Gynecol Reprod Biol. 2016;200:45-50. PMID: 26967346 DOI: 10.1016/j. ejogrb.2016.02.007

Sabatini L, Zosmer A, Hennessy EM, Tozer A, AlShawaf T. Relevance of basal serum FSH to IVF outcome varies with patient age. Reprod Biomed Online. 2008;17:10-9. PMID: 18616884 DOI: http://dx.doi.org/10.1016/S1472-6483(10)60287-8

Salinas M, López-Garrigós M, Gutiérrez M, Lugo J, Sirvent JV, Uris J. Achieving continuous improvement in laboratory organization through performance measurements: a seven-year experience. Clin Chem Lab Med. 2010;48:5761. PMID: 19919329 DOI: 10.1515/CCLM.2010.003

Sharma V, Allgar V, Rajkhowa M. Factors influencing the cumulative conception rate and discontinuation of in vitro fertilization treatment for infertility. Fertil Steril. 2002;78:40-6. PMID: 12095488 DOI: http://dx.doi.org/10.1016/S0015-0282(02)03160-6

Silberstein T, MacLaughlin DT, Shai I, Trimarchi JR, Lambert-Messerlian G, Seifer DB, Keefe DL, Blazar AS. Mullerian inhibiting substance levels at the time of HCG administration in IVF cycles predict both ovarian reserve and embryo morphology. Hum Reprod. 2006;21:159-63. PMID: 16123085 DOI: 10.1093/humrep/dei270

Strandell A, Bergh C, Lundin K. Selection of patients suitable for one-embryo transfer may reduce the rate of multiple births by half without impairment of overall birth rates. Hum Reprod. 2000;15:2520-5. PMID: 11098020 DOI: http://dx.doi.org/10.1093/humrep/15.12.2520 
Vaegter KK, Lakic TG, Olovsson M, Berglund L, Brodin $\mathrm{T}$, Holte J. Which factors are most predictive for live birth after in vitro fertilization and intracytoplasmic sperm injection (IVF/ICSI) treatments? Analysis of 100 prospectively recorded variables in 8,400 IVF/ICSI singleembryo transfers. Fertil Steril. 2017;107:641-648.e2. PMID: 28108009 DOI: 10.1016/j.fertnstert.2016.12.005

van Loendersloot LL, van Wely M, Limpens J, Bossuyt PM, Repping S, van der Veen F. Predictive factors in in vitro fertilization (IVF): a systematic review and meta-analysis. Hum Reprod Update. 2010;16:577-89. PMID: 20581128 DOI: $10.1093 /$ humupd/dmq015
Vermeulen RP, Jessurun GA, Peels HO, Jaarsma T, Zijlstra F. Clinical performance indicators for percutaneous coronary intervention. Crit Pathw Cardiol. 2008;7:126-32. PMID: 18520530 DOI: $10.1097 /$ HPC.0b013e318168af31

Wang YA, Healy D, Black D, Sullivan EA. Age-specific success rate for women undertaking their first assisted reproduction technology treatment using their own oocytes in Australia, 2002-2005. Hum Reprod. 2008;23:1633-8. PMID: 18441345 DOI: 10.1093/humrep/den135 